\title{
Selenium, selenoproteins and human health: a review
}

\author{
$\mathrm{KM} \mathrm{Brown}^{1 *}$ and JR Arthur ${ }^{2}$ \\ 'University of Aberdeen, Scotland: ${ }^{2}$ Rowett Research Institute, Aberdeen
}

\begin{abstract}
Selenium is of fundamental importance to human health. It is an essential component of several major metabolic pathways, including thyroid hormone metabolism, antioxidant defence systems, and immune function. The decline in blood selenium concentration in the UK and other European Union countries has therefore several potential public health implications, particularly in relation to the chronic disease prevalence of the Western world such as cancer and cardiovascular disease. Ten years have elapsed since recommended dietary intakes of selenium were introduced on the basis of blood glutathione peroxidase activity. Since then 30 new selenoproteins have been identified, of which 15 have been purified to allow characterisation of their biological function. The long term health implications in relation to declining selenium intakes have not yet been thoroughly examined, yet the implicit importance of selenium to human health is recognised universally.

Selenium is incorporated as selenocysteine at the active site of a wide range of selenoproteins. The four glutathione peroxidase enzymes (classical GPx1, gastrointestinal GPx2, plasma GPx3, phospholipid hydroperoxide GPx4)) which represent a major class of functionally important selenoproteins, were the first to be characterised.

Thioredoxin reductase (TR) is a recently identified seleno-cysteine containing enzyme which catalyzes the NADPH dependent reduction of thioredoxin and therefore plays a regulatory role in its metabolic activity.

Approximately $60 \%$ of Se in plasma is incorporated in selenoprotein $\mathrm{P}$ which contains 10 Se atoms per molecule as selenocysteine, and may serve as a transport protein for Se. However, selenoprotein-P is also expressed in many tissues which suggests that although it may facilitate whole body Se distribution, this may not be its sole function.

A second major class of selenoproteins are the iodothyronine deiodinase enzymes which catalyse the $5^{\prime} 5$-mono-deiodination of the prohormone thyroxine (T4) to the active thyroid hormone 3,3'5-triiodothyronine (T3).

Sperm capsule selenoprotein is localised in the mid-peice portion of spermatozoa where it stabilises the integrity of the sperm flagella.

Se intake effects tissue concentrations of selenoprotein W which is reported to be necessary for muscle metabolism.

It is of great concern that the health implications of the decline in Se status in the UK over the past two decades have not been systematically investigated. It is well recognised that dietary selenium is important for a healthy immune response. There is also evidence that Se has a protective effect against some forms of cancer; that it may enhance male fertility; decrease cardiovascular disease mortality, and regulate the inflammatory mediators in asthma. The potential influence of Se on these chronic diseases within the European population are important considerations when assessing Se requirement.
\end{abstract}

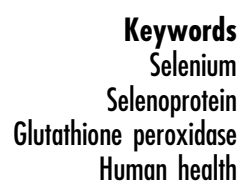

\section{Introduction}

In 1818 the Swedish chemist Jons Jacob Berzelius discovered selenium. He named it Selene after the Greek goddess of the moon. One hundred and forty years later, Schwarz and Foltz identified selenium as essential to animal health when they discovered that trace amounts protected against liver necrosis in vitamin $\mathrm{E}$ deficient rats ${ }^{1}$. Interest in the role of selenium in human health gathered momentum in the late 1960's, and investigations looked for human diseases similar to those of Se-responsive animal disorders ${ }^{2}$. Although 
selenium was identified as essential to human nutrition 42 years ago, a universal marker of daily requirement remains elusive. Research has extended our knowledge of the essential functional roles attributed to selenium, which have both short-, and long-term public health implications.

\section{Selenoproteins}

Selenium is an essential micronutrient of major metabolic significance. It is incorporated as selenocysteine at the active site of a wide range of proteins. Under physiological conditions the Se in selenocysteine is almost fully ionised and consequently is an extremely efficient biological catalyst ${ }^{3}$. It has been suggested that up to 100 selenoproteins may exist in mammalian systems ${ }^{4}$ of which up to 30 have been identified by 75 Se labelling in vivo 5 . To date 15 selenoproteins have been purified or cloned allowing further characterisation of their biological function. These include four glutathione peroxidase enzymes (classical GPx1, gastrointestinal GPx2, plasma GPx3, phospholipid hydroperoxide GPx4) which represent a major class of functionally important selenoproteins. The Se peroxidases are genetically, structurally and kinetically different yet have both common and individual functions ${ }^{3}$.

\section{Glutatbione peroxidase}

Classical glutathione peroxidase (GPx1) was the first selenoprotein, identified, and the strong linear relationship demonstrated between erythrocyte Se concentration and GSHPX activity provided us with the first functional biochemical marker of Se status ${ }^{6}$. GSHPx is present in the cell cytosol where it functions as an antioxidant by directly reducing $\mathrm{H}_{2} \mathrm{O}_{2}$, and phospholipase A2 cleaved lipid hydroperoxides ${ }^{6}$. It may also act as a storage vehicle for $\mathrm{Se}^{7}$ containing 4 selenocysteine residues in a tetrameric structure.

\section{Gastrointestinal glutatbione peroxidase}

Gastrointestinal glutathione peroxidase (GPx2) protects mammals from the toxicity of ingested lipid hydroperoxides $^{8}$. In animal studies, Se deficiency decreases the enzyme activity, but any effect on human GPx2 activity has not been reported. Gastrointestinal glutathione peroxidase is the most important selenoprotein antioxidant in the colon. Oxidative stress is a critical event in tumourogenesis. It is therefore likely that the antioxidant function of GPx2 will provide an early defence against colon cancer.

\section{Phospholipid bydroperoxide glutatbione peroxidase}

A membrane associated phospholipid hydroperoxide glutathione peroxidase (GPx4) has been identified which is directly responsible for the reductive destruction of lipid hydroperoxides 9 . The enzyme is a monomer and its activity is preserved in preference to GPx1 when dietary Se supply is low ${ }^{10}$. GPx 4 reacts with phospholid hydroperoxides as well as small soluble hydroperoxides $^{11}$, and is also capable of metabolising cholesterol and cholesterol ester hydroperoxides in oxidised low density lipoprotein. Consequently it is well recognised as essential to destruction of fatty acid hydroperoxides, which if not reduced to hydroxy fatty acids, will lead to uncontrolled free radical chain reactions that are deleterious to the integrity of membranes. In animal models, the amount of the protein GPx4 present in tissues does not exactly reflect the activity distribution. This may be a reflection of site specific Se dependent cell function, or differences in the level of factors which activate GPx4. The mechanism for the activation-inactivation of the enzyme is unknown, but the evidence of high activity in membranes of differentiating spermatogenic cells suggests a possible relationship between cell differentiation and peroxide levels ${ }^{12}$.

\section{Extracellular glutatbione peroxidase}

Extracellular GSHPx (GPx3) is another selenoprotein with antioxidant potential, but this may not be its main function in plasma. Hybridization studies show that GPx3 mRNA occurs in the renal proximal tubular epithelial cells ${ }^{13}$, and since the concentrations of $\mathrm{GSH}$ are high in the kidney, GPx3 may have a specific antioxidant function in renal tubules or extracellular spaces. However, other thiols such as thioredoxin can act as electron donor and support an antioxidant role for GPX3 in plasma ${ }^{14}$. Thioredoxin is a protein disulphide important to antioxidant defences and the regulation of cell growth.

\section{Thioredoxin reductase}

Thioredoxin reductase is a recently identified selenocysteine containing enzyme which catalyzes the NADPH dependent reduction of thioredoxin and therefore plays a regulatory role in its metabolic activity ${ }^{15}$. This discovery extends the role of Se to redox regulation. Since thioredoxin stimulates proliferation of normal and tumour cells, and is present in high concentrations in tumour cells $^{16}$, an enhanced TR activity may play an important role in the prevention of some forms of cancer.

\section{Selenoprotein $\boldsymbol{P}$}

Approximately $60 \%$ of Se in plasma is incorporated in selenoprotein $\mathrm{P}$ which contains 10 Se atoms per molecule as selenocysteine ${ }^{4}$. Extracellular GSHPx, and selenoprotein-P account for over $90 \%$ of plasma selenium and both may serve as a transport protein for $\mathrm{Se}^{17}$. However, selenoprotein-P is also expressed in many tissues and has been associated with cell membranes ${ }^{18}$ which suggests that although it may facilitate whole body Se distribution, this may not be its sole function, and it may serve as an antioxidant. 


\section{Iodotbyronine deiodinases}

A second major class of selenoproteins are the iodothyronine deiodinase enzymes which catalyse the 5'5mono-deiodination of the prohormone thyroxine $\left(\mathrm{T}_{4}\right)$ to the active thyroid hormone 3,3'5-triiodothyronine $\left(\mathrm{T}_{3}\right)$ and the conversion of inactive reverse $\mathrm{T} 3$ to 3 $3^{\prime}$ diiodothyronine. Thyroid hormones play a regulatory role in hepatic enzyme expression and neutrophil function. Consequently, in animal Se repletion studies, the lack of a relationship between the effect on drug metabolising enzymes and glutathione metabolism in the liver, neutrophil function, and changes in GSHPx activity prompted investigations into the association between Se and thyroid status ${ }^{19}$. Full activity of the thyroid hormones is dependent on the deiodination of thyroxine to triiodothronine $\left(\mathrm{T}_{3}\right)$. Most $\mathrm{T} 3$ is produced by the peripheral deiodination of $\mathrm{T}_{4}$ catalysed by the type 1 selenoenzyme iodothyronine $5^{\prime}$ deiodinase (IDI). There are three types of Se dependent iodothyronine deiodinase enzymes which function in specific tissues such as liver and brain, to maintain plasma and organ thyroid hormone homeostasis. Conversion of T4 to T3 can be assessed by monitoring the $\mathrm{T}_{3} / \mathrm{T}_{4}$ ratio in blood. There is a progressive reduction of this ratio with ageing which can be reversed with Se supplementation ${ }^{20}$. Thus the total $\mathrm{T}_{3} /$ total $\mathrm{T}_{4}$ ratio may serve as a functional marker of Se status in human studies. It may be logical to hypothesise that the regulatory role of thyroid hormones over cellular metabolic rate will be modulated by Se status, the requirement for which will increase in response to increased activity of the cell.

\section{Seleno-phosphate syntbetase}

Mechanisms for selenocysteine incorporation into functional selenoproteins involve an inorganic precursor, probably seleno-phosphate ${ }^{4}$. The fact that most dietary Se occurs in an organic form as either selenocysteine or selenomethione, suggests that the in vivo conversion to an inorganic precursor is an important regulator of Se bioavailability. This regulatory control may confer protection against excessive incorporation of Se into selenoproteins during protein synthesis, and may be of particular importance in preventing toxicity from excessive intakes.

\section{Sperm capsule selenoprotein GPx4}

GPx4 exists as a soluble peroxidase in spermatids but persists in mature spermatozoa as an enzymatically inactive, oxidatively cross-linked, insoluble protein ${ }^{21}$. Spermatozoa contain the highest concentrations of selenium of any mammalian tissue, with requirement increasing at the onset of spermatogenesis. At least 50 percent of the capsule material which supports the helix of mitochondria is $\mathrm{GPx} 4^{21}$. An insufficient Se supply impairs sperm mitochondrial capsule synthesis which affects sperm motility and may induce sterility. Selenium supplementation studies in infertile men increase seminal fluid Se concentration and improve sperm motility ${ }^{22}$. The potential influence of Se on the increasing male sterility in Europe is an important consideration when assessing Se requirement in men.

\section{Selenoprotien $\mathbf{W}$}

In animal studies, Se intake effects tissue concentrations of selenoprotein $\mathrm{W}$ which is reported to be necessary for muscle metabolism ${ }^{23}$. Skeletal muscle calcification in sheep and cattle, known as white muscle disease, is prevented by Se supplementation ${ }^{24}$. The importance of selenoprotein $\mathrm{W}$ to human skeletal muscle metabolism is not yet fully understood, but the recent cloning of its cDNA will enhance research into human muscle disease such as muscular dystrophies which have been shown to respond to Se supplementation. A myopathy caused by selenium deficiency, described as white muscle disease, has been reported in anorexia nervosa ${ }^{25}$.

\section{Dietary selenium and human requirement}

Selenium is present in soil and enters the food chain through plants. We obtain most of our dietary Se from bread, cereal, meat and poultry (Table 1). Tissue levels of Se are readily influenced by dietary intake which itself is governed by geographical differences in available selenium in soil. In general, soil concentration of available Se is low in Europe. Bioavailability of Se is also low and has decreased further as a consequence of increasing acid rain and use of excessive fertilisation. Generally speaking, human blood Se levels follow the same geographical pattern around the world as those of livestock in the same regions ${ }^{26}$. Although Se deficiency in animals has long been recognised, obvious clinical signs of human Se deficiency are rare. The exception is in areas of North-East China with very low soil Se where an endemic, fatal cardiomyopathy, Keshan disease, was found to respond to Se supplementation. Asymptomatic low Se status is also reported in New Zealand, Finland and areas of the Eastern United States where government mandates have initiated

Table 1 Mean selenium concentrations in various European food sources

\begin{tabular}{lc}
\hline Food & $\begin{array}{c}\text { Mean selenium } \\
\text { concentration } \mu \mathrm{g} / 100 \mathrm{~g}\end{array}$ \\
\hline Milk & 1.5 \\
Beef & 7.6 \\
Pork & 14.0 \\
Lamb & 3.8 \\
Liver & 42.0 \\
Kidney & 145.0 \\
Fish & 16 \\
Fruit & 1 \\
Vegetables & 2 \\
Cereals & 11 \\
Bread & 4.5 \\
Brazil nuts & 254 \\
\hline
\end{tabular}


various Se supplementation schemes. These involve either enriched food products, soil fertiliser or direct supplementation regimens.

There has been a substantial fall in Se intake in the UK and other European Union countries, largely because of the decrease in imports of Se rich wheat from North America. This is reflected in the serum Se concentrations across Europe which range from $0.63-1.69 \mu \mathrm{mol} / \mathrm{L}^{27,28}$. Values in areas of Greece, West Germany, Sweden, Spain, Denmark, France, Belgium, the Netherlands, and the UK are reported to be lower than the $1.2 \mu \mathrm{mol} / \mathrm{L}$ required for optimal (defined as maximal) GPx1 activity (Table 2). In Finland, increasing dietary selenium intakes using selenium enriched fertilizers, from an average of $25 \mu \mathrm{g} /$ day to $70 \mu \mathrm{g} /$ day had the effect of raising serum selenium concentrations from $0.63 \mu \mathrm{mol} / \mathrm{L}$ in the 1970's to $1.42 \mu \mathrm{mol} / \mathrm{L}$ in the 1990's. The health implications of this change have not been reported.

Selenium presents a nutritional conundrum because it is both essential and highly toxic. Measurement of glutathione peroxidase activity has been used as a marker of adequacy of intake because activity of the enzyme is proportional to dietary intake. Comparing dietary intakes from different geographical regions where Se soil concentrations are very different has provided information on the intake required to maximise glutathione peroxidase activity. In the Se depleted region of North east China Se intakes of below $11 \mu \mathrm{g}$ Se/day are causally linked to the endemic juvenile cardiomyopathy, Keshan disease. In a study of adult men living in this area of China who were supplemented with $30 \mu \mathrm{g}$ of selenomethionine/day plasma GPx3 activity increased to what was considered maximum activity ${ }^{29}$. Dietary intakes of $41 \mu \mathrm{g} \mathrm{Se} /$ day were subsequently considered sufficient to meet the Se requirement of men with an average body weight of $60 \mathrm{~kg}$. This data was used as the basis on which to make recommendations. The Scientific Committee for Food has proposed that $40 \mu \mathrm{g} \mathrm{Se} / \mathrm{day}$ is a sufficient average intake for the European Community ${ }^{30}$. Discussions on Se requirements by FAO, IAEA, and WHO suggest intakes of $40 \mu \mathrm{g}$ and $30 \mu \mathrm{g}$ are sufficient to meet normal requirements of adult males and females respectively $^{31}$. Such judgements of requirement are based on Se

Table 2 Serum selenium concentrations of several European populations

\begin{tabular}{lc}
\hline Country & $\begin{array}{c}\text { Serum selenium } \\
\text { concentration } \mu \mathrm{mol} / \mathrm{L}(\mathrm{SD})\end{array}$ \\
\hline UK & $1.01(0.16)$ \\
Greece & $0.79(0.17)$ \\
West Germany & $0.81(0.12)$ \\
Sweden & $1.02(0.13)$ \\
Spain & $1.1(0.17)$ \\
Denmark & $0.98(0.18)$ \\
France & $1.0(0.18)$ \\
Belgium & $1.07(0.16)$ \\
Netherlands & $1.17(0.18)$ \\
\hline
\end{tabular}

intakes sufficient to allow expression of two-thirds of erythrocyte GPx1 activity, with lower limits of safe population intakes calculated from basal metabolic rate. These values do not meet the recommendation that selenium intake should approximate $1 \mu \mathrm{g} / \mathrm{kg}$ body weight. Since supra-nutritional levels of Se intake are required to reduce the incidence of animal and human cancers, it is likely that maintaining the activities of known selenoproteins is not the mechanism by which se acts since it appears to be saturated at normal nutritional intakes. It is 30 years since glutathione peroxidase activity was identified as a functional marker of Se status. Since this time many more selenoproteins have been identified as essential to major metabolic pathways. Consequently, it may be no longer appropriate to rely on glutathione peroxidase activity as an index of adequacy of Se intake. Recommendations are routinely based on intakes which prevent overt symptoms of deficiency. These do not take account of the many more recently identified functional roles of Se. Nor do they address the potential functional effects of chronic marginal intakes of Se which may culminate in chronic disease such as cancer, cardiovascular disease, and increased susceptibility to viral infection. It is of course very difficult to make recommendations, since specific sensitive biochemical markers of functional Se status are not yet available. Moreover, there is growing emphasis on assessing nutrient status with functional rather than static biomarkers.

\section{Selenium and health}

The health implications of the decline in Se status in the UK over the past two decades have not been systematically investigated. Dietary intake of Se in the UK is now reported to be only half the UK Reference Nutrient Intake (RNI) of $1 \mu \mathrm{g} \mathrm{Se} / \mathrm{kg}$ body weight $\mathrm{w}^{32,33,34}$. Our own research highlights the relatively poor Se status of a UK population ${ }^{35}$ with plasma Se levels lower than many other European countries ${ }^{28}$, and levels in erythrocytes half the value previously reported for UK populations ${ }^{36,37}$. Se supplementation of our volunteers with $50 \mu \mathrm{g} \mathrm{Se} /$ day as sodium selenite or selenomethionine, increased activities of Se dependent peroxidases in plasma and blood cells, indicating that functional Se status may be improved by nutritional means ${ }^{35}$. The different and contrasting effects that Se supplementation had on white blood cell and platelet selenoenzyme activities, and the fact these were seen in only $60-70 \%$ of subjects, may be indicative of a difference in metabolic need regulated at the level of Se dependent cell function. Transient spikes in activity of GPx4 an extremely stable enzyme which is preferentially spared in seleniumdepleted animal models, may indicate that it is a marker of increased selenium requirement in humans. Lipid peroxidation is only inhibited by GPx4 if membranes contain sufficient vitamin E, consistent with the proposed 
synergism between the two antioxidant activities ${ }^{38}$. Subjects who do not respond to Se supplementation may have either sufficient basal levels of expression, an inability to express the protein, or an impaired ability to activate the protein.

\section{Immune function}

Although the mechanisms involved have yet to be fully elucidated, it is well established that dietary selenium is important for a healthy immune response ${ }^{39}$. The effects of Se deficiency can include reduced T-cell counts, impaired lymphocyte proliferation and responsiveness ${ }^{40}$. Dietary supplementation of humans with $200 \mu \mathrm{g}$ of sodium selenite enhances T-lymphocyte immune responses ${ }^{41}$. A progressive decline in plasma Se has been widely reported in adult respiratory distress syndrome (ARDS) and AIDS patients, and approximately parallels T-cell loss or stage of HIV infection. It is particularly noticeable at the terminal stages of disease where Se deficiency is now considered a classical symptom/sign of end stage. There is an extremely high turnover of CD4 T-cells in AIDS, with billions of new cells lost and replaced daily. The constant formation of new cells to replace those lost requires an extremely efficient and effective Se supply to keep up with the high demand in active lymphocytes. Sub-clinical requirement for Se by lymphocytes and granulocytes will clearly not be as great when the immune system is not overly stressed. After selenium supplementation, the range of variation in \% change in selenoenzyme activity between individuals may reflect the tocopherol status of the blood cells. Lymphocytes and granulocytes can contain up to 35 times more alpha-tocopherol than red blood cells or platelets, probably reflecting preferential mobilization to cells with different metabolic need. All of these cells are capable of increasing their metabolic activity as a function of their immunosuppressive role. In doing so, membrane sensitive oxidases are generated which oxidise NADH and NADPH thereby increasing $\mathrm{O}_{2}$ utilization and its subsequent reduction to reactive oxygen species (ROS). Tocopherol and Se supply to these cells is therefore essential to provide control over their functional generation of excessive ROS.

\section{Cancer}

There is strong evidence that Se has a protective effect against some forms of cancer. In a recent study involving 1312 patients supplemented with $200 \mu \mathrm{g}$ Se daily, the incidence of prostate, colon and lung cancers was decreased by 63,58 , and $46 \%$ respectively ${ }^{42}$. The mechanism of action of the chemoprotective effects are not known, but may be mediated through the two major Se dependent redox systems in the cell.

The principle cell types that mediate the eradication of toxins and mutated cells in the circulation, are the Tlymphocytes, the synthesis and activation of which may be Se dependent. It appears that the CD4 gene Open
Reading Frame may contain the same selenocysteine insertion sequences as mammalian selenoprotein $\mathrm{P}^{43}$, thus Se may be important to T-cell protein synthesis and therefore normal cell function. This may be of relevance to the observations of Clark et $a l^{42}$ in relation to Se supplementation and cancer aetiology because Tlymphocytes are the principal cell types that eradicate tumour cells. Thioredoxin reductase has recently been identified and purified from human T-cells ${ }^{43}$, and may be important in reducing thioredoxin enhanced tumour cell growth.

\section{Cardiovascular disease}

Low blood Se concentrations have been associated with increased cardiovascular disease mortality. This may be a reflection of sub-optimal GPx4 activity in the prevention of LDL oxidation, with subsequent uptake by endothelial cells and macrophages in arterial blood vessels. Heart disease mortality declined by an average 61\% in Finland between 1972 and 1992. The decline has been attributed to major lifesyle changes, the most important reported as a $4 \%$ fall in energy consumed from fat, with an associated lowering of blood cholesterol concentration. It is highly likely, that the concomitant 3 fold increase in dietary Se intake as a consequence of Se enrichment of fertilizer, since 1985 will also have contributed to lower heart disease mortality reported in 1992.

\section{Astbma}

Evidence of an extended role of GPx 4 beyond that of an antioxidant comes from the work of Weitzel and Wendel $(1993)^{44}$, who have demonstrated that GPx4 regulates the activity of lymphocyte 5-lipoxygenase, and Steinhilber D. et $a l^{45}$, who show that GPx 4 supresses 5-lypoxygenase activity in lymphocytes and granulocytes. Consequently, GPx 4 may have a regulatory role in the inflammatory response through suppression of lypoxygenase catalysed leukotriene biosynthesis from arachidonic acid. Moreover, it has been hypothesised that vitamin E may have a regulatory influence over leukotriene biosynthesis as a substrate for both n-6 and n-3 unsaturated fatty acid desaturase enzymes ${ }^{46}$. This indication that functional selenium and vitamin E status may influence leucotriene metabolism has important implications in relation to chronic inflammatory disease, particularly asthma which is now the most prevalent chronic inflammatory condition in childhood, and has doubled over the last 20 years in the $\mathrm{UK}^{47}$. There is a dramatic rise in the prevalence of asthma in the UK which mirrors the dramatic decline in blood Se concentration. This situation has the potential to exacerbate the imbalance in $\mathrm{n} 6 / \mathrm{n} 3$ fatty acid status already recognised as involved in the pathogenesis of the disease. Moreover, Se status is decreased in patients with asthma, as is activity of glutathione peroxidase in platelets and erythrocytes. There is an associated marked oxidant/antioxidant imbalance in the blood of asthmatics, 
which reflects poor antioxidant status and enhanced inflammatory mediated oxidative stress.

\section{Summary}

An adequate dietary supply of Se is essential for selenoenzyme activity. Consequently, the decreased intake of Se and lowered Se status of several European populations is of concern since it may result in sub-optimal selenoenzyme activity which may have deleterious long term health implications.

Many new functional roles for selenium are being identified that still require to be elucidated. This should not however, detract from further investigation into the long term implications of lowered Se status in Europe, the health implications of which have not been systematically investigated.

Selenium is an integral component of at least three major metabolic systems essential for normal cell metabolism ${ }^{48}$. There are no classical overt signs of clinical deficiency in European populations, but there are in populations of North East China where a fatal endemic cardiomyopathy is associated with low dietary Se supply and blood concentration, and is successfully treated by Se supplementation. What is astonishing is that the disease is evident at blood Se levels which are only just lower than in some European subjects. What may be important in terms of the functional role of selenium is the range in blood Se concentrations of our populations.

Blood selenium concentration data does reflect dietary intake but tells us nothing of its functional significance, particularly in relation to its important role in thyroid hormone metabolism, antioxidant defence and redox systems, and the immune response, in particular lymphocyte and neutrophil function. It is also evident from clinical studies that increasing se intake decreases infection rate, and susceptibility to viral mutation, which might increase the virulence of the pathogen. These biological requirements, and the chemopreventive potential of Se, means there is an urgent need to establish valid biomarkers in terms of functional requirement and adequacy of intake.

Individuals may have differing metabolic needs for selenium regulated at the level of Se dependent cell function, for example in relation to immuno-responsive roles. Of course there will be substantial genetic variation between individuals which is why we must examine nutrient gene interactions in the future. We must also consider other factors such as the regulatory role of alpha-tocopherol, and evaluate the most recent functional roles attributed to selenoproteins as potential markers of requirement. The large intra-individual and inter-individual variations in selenoenzyme activity suggests that it may not on its own be a valid biomarker of functional Se status. Only future research can provide a truly functional measure of adequacy of Se intake.

\section{References}

1 Schwartz K, Foltz SM. Se as an integral part of factor 3 against necrotic liver degeneration. J. Mountain Chemical Society 1957; 79: 3292-3.

2 Combs SB, Combs GF. The role of selenium in Nutrition. New York: Academic Press Inc, 1986.

3 Arthur JR, Brown KM, Fairweather-Tait SJ, Crews HM. Dietary selenium: why do we need it and how much is enough? Nutrition \& Food Science 1997; 6: 225-8.

4 Burk RF, Hill KE. Regulation of Selenoproteins. Annual Review of Nutrition 1993; 13: 65-81.

5 Evenson JK, Sunde RA. Selenium incorporation into selenoproteins in the Se-adequate and Se-deficient rat. Proceedings of Society for Experimental Biology and Medicine 1988; 187: $169-80$.

6 Rotruck JT, Pope AL, Ganther HE, Swanson AB, Hafeman DG, Hoekstra WG. Selenium: biochemical role as a component of glutathione peroxidase. Science 1973; 179: 588-90.

7 Burk RF. Molecular biology of selenium with implications for its metabolism. FASEB 1991; 55: 2279.

8 Chu FF, Doroshow JH, Esworthy RS. Expression, characterization and tissue distribution of a new cellular selenium dependent GSHPx. J. Biol. Chem. 1993; 268: 2571-6.

9 Ursini F, Maiorino M, Gregolin C. The selenoenzyme phospholipid hydroperoxide glutathione peroxidase. Biochim. Biophys. Acta. 1985; 839: 62-70.

10 Bermano G, Nicol F, Dyer JA, Sunde RA, Beckett GJ, Arthur JR, Hesketh JE. Tissue-specific regulation of selenoenzyme gene expression during selenium deficiency in rats. Biochem. J. 1995; 311: 425-30.

11 Weitzel F, Ursini F, Wendel A. Phospholipid Hydroperoxide Glutathione Peroxidase in Various Mouse Organs During Selenium Deficiency and Repletion. Biochimica-etBiophysica-Acta. 1990; 1036: 88-94.

12 Calvin HI, Grosshans K, Musicant-Shikora SR, Turner SI. A developmental study of rat sperm and testis selenoproteins. J. Reprod. Fertil. 1987; 81: 1-11.

13 Avissar N, Ornt DB, Yagil Y, Horowitz S, Watkins RH, Kerl EA, Takahashi K, Palmer IS, Cohen HJ. Human Kidney Proximal Tubules Are the Main Source of Plasma Glutathione Peroxidase. Am. J. Physiol. 1994; 266: C367-75.

14 Holmgren A. Thioredoxin and glutaredoxin systems. J. Biol. Chem. 1989; 264: 13963-6.

15 Sun Q-A, Wu Y, Zappacosta F, Jeang KT, Lee BJ, Hatfield DL, Gladyshev VN. Redox Regulation of Cell Signaling by Selenocysteine in Mammalian Thioredoxin Reductases. $J$ Biol. Chem. 1999; 274: 24522-30.

16 Holmgren A. Thioredoxin and thrioredoxin reductase. Methods Enzymol. 1995; 252: 199-208.

17 Mostenbocker MA, Tappel AL. A selenocysteine containing seleniujm transport protein in rat plasma. Biochim. Biophys. Acta. 1982; 719: 147-53.

18 Burk RF, Hill KE. Selenoprotein P: A selenium rich extra cellular glycoprotein. J. Nutr. 1994; 124(10): 1891-7.

19 Arthur JR, Nicol F, Becket GT. Selenium deficiency, thyroid hormone metabolism and thyroid hormone deiodinases. Am. J. Clin. Nutr. 1993; s57: 5236-9.

20 Olivieri O, Girrelli D, Azzini M. Low selenium status in the elderly influences thyroid hormones. Clin. Sci. 1995; 89: 637-42.

21 Ursini F, Heim S, Kiess M, Maiorino M, Roveri A, Wissing J, Flohe L. Dual function of the selenoprotein PHGPx during sperm maturation. Science 1999; 285: 1393-6.

22 Venzina D, Mauffette F, Roberts KD. selenium vitamin E supplementation in infertile men. Biol. Trace Elem. Res. 1996; 53: $65-83$.

23 Yeh JY, Vendeland SC, Gu QP. Dietary selenium increases selenoprotein $\mathrm{W}$ levels in rat tissues. J. Nutr. 1997; 127: $2165-72$. 
24 Westermarck HW. Selenium in long term feeding and the frequency of white muscle disease in cattle in Finland during the years 1978-1985. Journal of Agricultural Science Finland 1987; 59: 47-50.

25 Ishihara H, Kanda F, Matsushita T, et al. White muscle disease in humans: myopathy caused by selenium deficiency in anorexia nervosa under long term total parenteral nutrition. J. Neurol. Neurosur PS 1999; 67: 829-30.

26 Casey CE, Guthrie BE, Friend GM. Selenium in human tissues from New Zealand. Arch. Environ. Health 1982; 37 : $133-5$.

27 Thorling EB, Overvad K, Heerfordt A. Serum selenium in Danish blood bank donors. Biol. Trace Elem. Res. 1985; 8: $65-73$.

28 Alfthan G, Neve J. Reference values for serum selenium in various areas - Evaluated according to the TRACY protocol. Journal Trace Elements in Medicine and Biology 1996; 10: $77-87$.

29 Levander OA. Scientific rationale for the 1989 recommended dietary allowance for selenium. Journal of American Dietetic Association 1991; 91: 1572-6.

30 WHO Food and Agriculture Organisation, International Atomic Energy Agency expert group. Trace elements in buman nutrition and health. Geneva: WHO, 1996.

31 Levander O, Whanger P. Deliberations and Evaluations of the Approaches, Endpoints and Paradigns for Selenium and Iodine Dietary Recommendations. J. Nutr. 1996; 126: $2427 \mathrm{~s}-34 \mathrm{~s}$.

32 Molnar J, MacPherson A, Barclay I, Molnar P. Selenium content of convenience and fast foods in Ayrshire, Scotland International. Journal Food Sciences and Nutrition 1995; 46: $343-52$.

33 Shortt CT, Duthie GG, Robertson JD, Morrice PC, Nicol F, Arthur JR. Selenium studies of a group of Scottish adults. Eur. J. Clin. Nutr. 1997; 51: 1-5.

34 MAFF Joint Food Safety and Standards Group. Food Surveillance Information Sheet 1997 No 127.

35 Brown KM, Pickard K, Nicol F, Duthie G, Beckett G, Arthur JR. Effects of organic and inorganic selenium supplementation on selenoenzyme activity in blood lymphocytes, granulocytes, platelets and erythrocytes. Clin. Sci. 2000; 98: 593-9.

36 Diplock AT. Indexes of Selenium Status in Human Populations American. J. Clin. Nutr. 1993; 57: S256-8.

37 Rayman MP. Dietary selenium: Time to act - Low bioavailability in Britain and Europe could be contributing to cancers, cardiovascular disease, and subfertility. Br. Med. J. 1997; 314: 387-8.

38 Roveri A, Maiorino M, Ursini F. Enzymatic and immunological measurements of soluble and membrane- bound phospholipid-hydroperoxide glutathione peroxidase. Methods Enzymol. 1994; 233: 202-12.

39 Taylor EW. Selenium and cellular immunity - Evidence that selenoproteins may be encoded in the +1 reading frame overlapping the human CD4, CD8, and HLA-DR genes. Biol. Trace Elem. Res. 1995; 49: 85-95.

40 Kiremidjian-Schumacher L, Roy M, Wishe HI, Cohen MW, Strotzky G. Supplementation with selenium and human immune cell functions. 2. Effect on cytotoxic lymphocytes and natural killer cells. Biol. Trace Elem. Res. 1994; 41: 115-27.

41 Roy M, Kiremidjian-Schumacher L, Wishe H, Cohen MW, Stotzy G. Supplementation with selenium and human immune cell functions 1. Effect on lymphocyte proliferation and interleukin 2 receptor expression. Biol. Trace Elem. Res. 1994; 41: 103-14.

42 Clark LC, Combs GF, Turnbull BW, Slate EH, Chalker DK, Chow J, Davis LS, Glover RA, Graham GF, Gross EG, Krongrad A, Lesher JL, Park HK, Sanders BB, Smith CL, Taylor JR. Effects of selenium supplementation for cancer prevention in patients with carcinoma of the skin: A randomized controlled trial. J. Am. Med. Assoc. 1996; 276: 1957-63.

43 Gladyshev VN, Jeang KT, Stadtman TC. Selenocysteine, identified as the penultimate C-terminal residue in human T-cell thioredoxin reductase, corresponds to TGA in the human placental gene. Proc. Natl. Acad. Sci. U.S.A. 1996; 93: 6146-51.

44 Weitzel F, Wendel A. Selenoenzymes Regulate the Activity of Leukocyte 5-Lipoxygenase via the Peroxide Tone. J. Biol. Chem. 1993; 268: 6288-92.

45 Steinhilber D, Herrmann T, Rothe HJ. Selenium dependent peroxidases suppress 5-lypoxygenase activity in B-lymphocytes and immature myeloid cells. Eur. J. Biochem. 1996; 242: 90-7.

46 Infante JP. Vitamin E and selenium participation in fatty acid desaturation: A proposal for an enzymatic function of these nutrients. Mol. Cell Biochem. 1986; 69(2): 93-108.

47 Seaton A, Godden DJ, Brown K. Increase in asthma: A more toxic environment or a more susceptible population. Thorax 1994; 49: 171-4.

48 Arthur JR. Functional indicators of iodine and selenium status. Proceedings of the Nutrition Society 1999; 58: 507-12. 